\title{
The effect of fatigue crack rate on the heat dissipation in metals under mixed-mode loading
}

\author{
Oleg Plekhov \\ Institute of Continuous Media Mechanics of the Ural Branch of Russian Academy of Science, Perm, Russia \\ poa@icmm.ru
}

\author{
Aleksei Vshivkov \\ Institute of Continuous Media Mechanics of the Ural Branch of Russian Academy of Science, Perm, Russia \\ vshivkov.a@icmm.ru
}

\begin{abstract}
The study of energy balance at crack tip could give comprehensive information for estimation of durability and reliability cracked construction under cyclic loading. The first step for estimation energy balance is monitoring of dissipated part of energy (heat generation). One of the most effective techniques for investigation of temperature evolution on the surface of deformed body is infrared thermography. The work is devoted to the experimental study of energy dissipation in cracked samples made of titanium Grade 2 under multiaxial cyclic loading. To explain the experimentally obtained correlation between fatigue crack rate and power of heat dissipation we developed a phenomenological model describing the evolution of plastic work at the crack tip under arbitrary loading. The theoretical and experimental data about heat dissipation exhibit a good quantitative agreement.
\end{abstract}

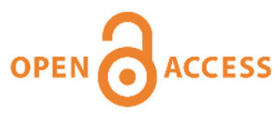

Citation: Plekhov, O., Vshivkov, A., The effect of fatigue crack rate on the heat dissipation in metals under mix mode loading, Frattura ed Integrità Strutturale, 50 (2019) 1-8.

Received: 26.12 .2018

Accepted: 08.07.2019

Published: 01.10.2019

Copyright: (C) 2019 This is an open access article under the terms of the CC-BY 4.0, which permits unrestricted use, distribution, and reproduction in any medium, provided the original author and source are credited.

KEYWORDS. Energy dissipation; Crack propagation; Infrared thermography; Mixed-mode loading.

\section{INTRODUCTION}

he most frequently used technique for estimation of lifetime of cracked samples is based on the correlation between stress intensity factor and fatigue crack rate (Paris `law). Paris law is results treatment of many experimental data in framework of elastic solution of crack propagation problem. As a consequence, this law doesn `t explain the physical nature of the process of crack propagation in metals and initiates a lot of scientific discussions and attempts to derive alternative description of crack propagation law.

Such alternative laws of crack propagation were proposed by many authors based on correlations of the fatigue crack growth rate and mechanical-structural parameters describing the failure process at crack tip. For instance, the intensity of plastic 
deformation, the Rise integral, the sizes of plastic deformation zone at crack tip, the dissipated energy were used as the crack propagation control parameters [1-4].

To derive a crack propagation law valid for arbitrary loading conditions we have to consider an energy concept of crack propagation. The current state of development of experimental mechanics allows one to monitor the dissipated part of energy with very high precision using infrared thermography [5-7]. The main problem of application of thermography technique is caused by the uncertainty of the solution to the inverse thermal problem [8]. Few years ago, we proposed an effective chip solution for the problem. We developed an additional system for direct monitoring of a heat flow [9].

To treat the experimental data we need a simple description of elasto-plastic deformation in process zone valid for arbitrary (multiaxial) loading conditions. Following by idea [10], we proposed a model of energy dissipation at crack tip. The key point of this approach is a hypothesis of the link between the elastic and elasto-plastic solutions at the fatigue crack tip proposed by Dixon [11]. In the framework of the model we divided the dissipated energy into two parts corresponding to reversible (cyclic) and monotonic plastic zones.

Analysis of this approximation has shown zero effect of fatigue crack advance on the energy dissipation into cyclic plastic zone. This dissipation is a function of spatial size of a cyclic plastic zone and characteristic size of the yield surface. Under isotropic hardening, the change of the applied stress amplitude leads to the change of characteristic size of the yield surface and, as consequence, to the heat dissipation at constant crack rate. The dissipation in monotonic plastic zone is a function of both crack rate and characteristic size of the yield surface. This part of the model gives correlation between fatigue crack rate and power of heat dissipation $[4,7]$.

The previous authors' investigations were focused on crack growth problems under an opening or mode I mechanism [9] and a relationship was proposed for the growth rate of a fatigue crack based on an analysis of the energy balance at its tip. However, most structures are failed due to mixed mode loading. Many uniaxial loaded materials, structures and components often contain randomly oriented defects and cracks which develop a mixed mode state by rotation of their orientation with respect to the loading axis. For example mixed mode I/II cyclic deformation at the tip of a short kinked inclined crack with frictional surfaces as discussed in the following [12-15].

In this work, we verify the main hypothesis used for our phenomenological description of fatigue crack propagation law and verify the approach for fatigue cracks titanium Grade 2 samples subject to the multiaxial cyclic loading.

\section{ENERGY DISSIPATION AT THE CRACK TIP UNDER CYCLIC LOADING}

$\mathrm{I}$

$\mathrm{n}$ the previous work [9] we obtained the relation to calculate the energy of plastic deformation as a consequence, energy dissipation at fatigue crack tip:

$$
U_{p}^{t o t}=W_{1}\left(A_{\tau}^{2}\right)+W_{2}\left(A_{\tau}^{2}\right) \frac{d a}{d N}
$$

where $A_{\tau}-$ stress amplitude.

Terms in Eqn. (1) correspond to reversible (cyclic) and monotonic plastic zones. Analysis of this approximation has shown that energy dissipation in a cyclic plastic zone is independent of crack growth. This dissipation is fully determined by the spatial size of a cyclic plastic zone and the characteristic diameter of the yield surface. For isotropic hardening materials, the change of the applied stress amplitude leads to the change in the characteristic diameter of the yield surface and, as consequence, to the energy dissipation at a constant crack rate. Dissipation in the monotonic plastic zone is a function of both crack rate and characteristic diameter of the yield surface.

It was shown by Short [1], that crack growth can be determined by heat generation:

$$
\frac{d a}{d N}=\frac{W^{p}-\Phi}{\Gamma-J}
$$

Where $W^{p}$ - hysteresis energy rate, $\Phi$ - heat generation rate, $\mathrm{J}$ - fracture energy added to $\mathrm{V}$ per unit crack advance, $\Gamma$ - fracture energy required per unit crack advance (original notation are used).

We can write the equation for heat generation from (2): 


$$
\Phi=W^{p}-(\Gamma-J) \frac{d a}{d N}
$$

In the general case, Eqns. (1) and (3) are same. The calculation of analytical relations for $W_{1}, W_{2}$ can be carried out similarly to the monotonic loading. It allows us to predict the existence of peculiarities of energy dissipation at the crack tip reported in [17] for multiaxial loading. Thus, we have an analytical equation for calculating heat generation, obtained in two different ways. The relation (1) obtained based on Dixon's hypothesis (Eqn. (4)) [11] about relation between elastic and plastic deformation.

$$
\varepsilon_{\mathrm{ij}}^{e f}=\left(\frac{E}{E_{s}}\right)^{\frac{1}{2}} \varepsilon_{i j}^{e l}
$$

where $E$ - the Young's modulus, $E_{S}$ - secant plasticity modulus.

\section{NUMERICAL SIMULATION OF STRAIN FIELD AT THE CRACK TIP}

7 he check an applicability of Dixon's hypothesis (Eqn. (1)) about relation between elastic and plastic deformation was carried out by numerical simulation of strain field at the crack tip. There was considered a two-dimensional model of biaxial loading. Half of a sample with symmetry of inner boundary was studied. In calculation the plane strain assumption was adopted. A square grid with a concentration in the region near the crack tip was used. An element size at crack tip was up to $5 \mathrm{e}-6 \mathrm{~m}$. To describe plastic deformation the isotropic hardening function was used which was obtained from the approximation of experimental data. The applied load corresponds to the experimental values. A crack path was taken from the digital image correlation data. The system of equations for modeling of a strain field near the crack tip has the following form:

$$
\begin{aligned}
& \nabla \cdot \boldsymbol{\sigma}=\mathbf{0}, \\
& \boldsymbol{\sigma}=\mathbf{C}:\left(\boldsymbol{\varepsilon}-\boldsymbol{\varepsilon}^{p}\right), \\
& \boldsymbol{\varepsilon}=\frac{1}{2}\left[\bar{\nabla} \mathbf{u}+\bar{\nabla} \mathbf{u}^{T}\right], \\
& \boldsymbol{\varepsilon}^{p}=\lambda \frac{\partial F}{\partial \boldsymbol{\sigma}},
\end{aligned}
$$

where $\sigma$-stress, $\varepsilon$ - strain, $\mathrm{u}$ - displacement, $\lambda$ - hardening parameter, $\mathrm{F}=\sigma_{\mathrm{m}}-\sigma_{\mathrm{ys}}, \sigma_{\mathrm{ys}}=\sigma_{\mathrm{ys} 0}+\sigma_{\mathrm{h}}\left(\varepsilon_{\mathrm{pe}}\right), \sigma_{\mathrm{h}}\left(\varepsilon_{\mathrm{pe}}\right)$ - hardening function. A numerical simulation of plastic deformation near the crack tip has been carried out. The estimation of the plastic strain field was carried out using the elastic solution and the Eqn. (4) for the strain components $\varepsilon_{11}, \varepsilon_{12}, \varepsilon_{22}$, maximum shear strain $\gamma$, effective plastic deformation $\varepsilon_{\mathrm{pe}}$ :

$$
\varepsilon_{p e}=\frac{\sqrt{2}}{3}\left(\left(\varepsilon_{11}-\varepsilon_{22}\right)^{2}+\left(\varepsilon_{11}-\varepsilon_{33}\right)^{2}+\left(\varepsilon_{33}-\varepsilon_{22}\right)^{2}+\frac{3}{2}\left(\varepsilon_{12}^{2}+\varepsilon_{13}^{2}+\varepsilon_{23}^{2}\right)\right)^{1 / 2} .
$$

Numerical and theoretical calculations were made for four biaxial coefficients $(\eta=0,0.5,0.7,1)$. The characteristic distributions of normal to the crack path strain component and second strain invariant are shown in Figs. 1, 2. To compare the theoretical and calculated results, point-by-point error was calculated for different strain levels corresponded to the applied load (Fig. 3). The loading levels correspond to the crack growth rate 1e-8 - 1e-3m/cycle. 


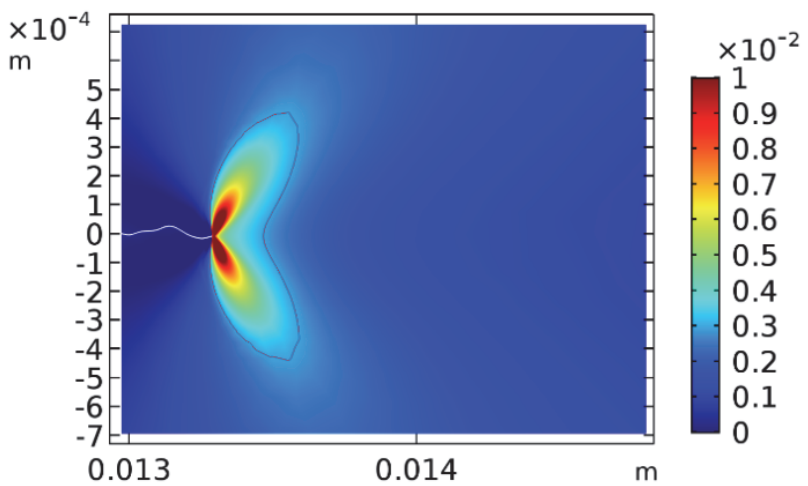

(a)

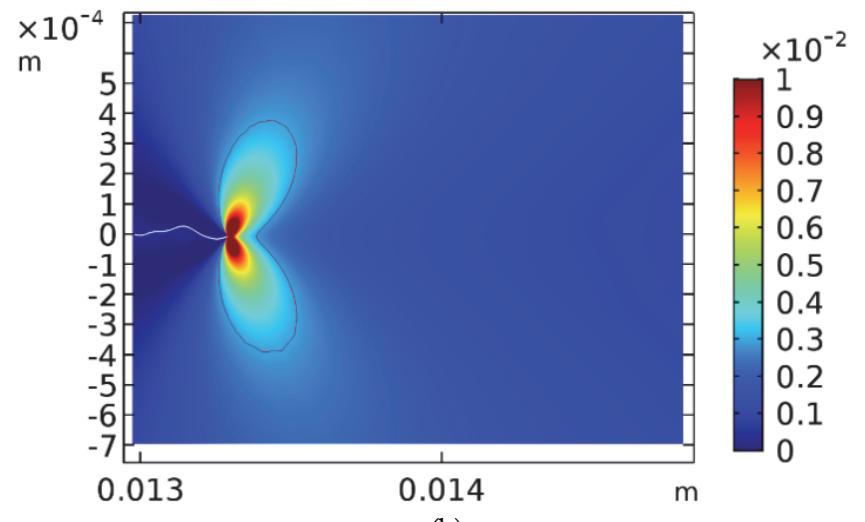

(b)

Figure 1: Plastic strain ( $\eta=0$, component $\varepsilon_{22}$ ): (a) numerical simulation; (b) analytic solution by Eqn. (4).

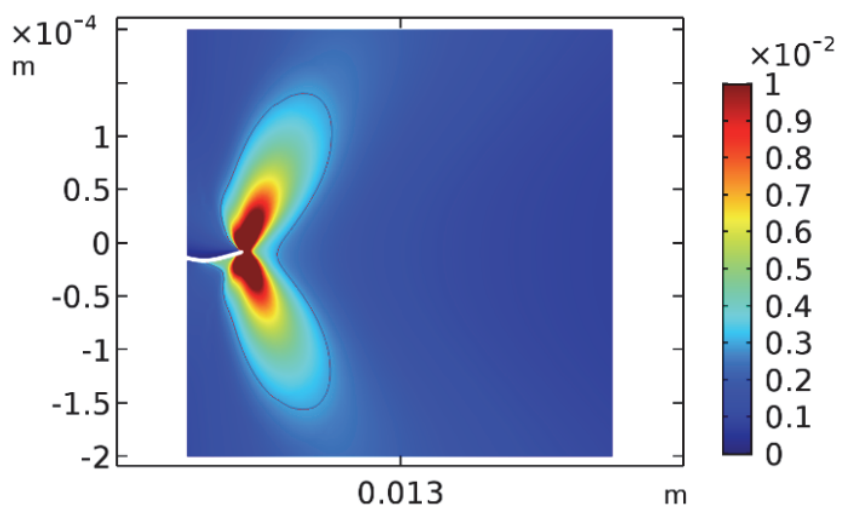

(a)

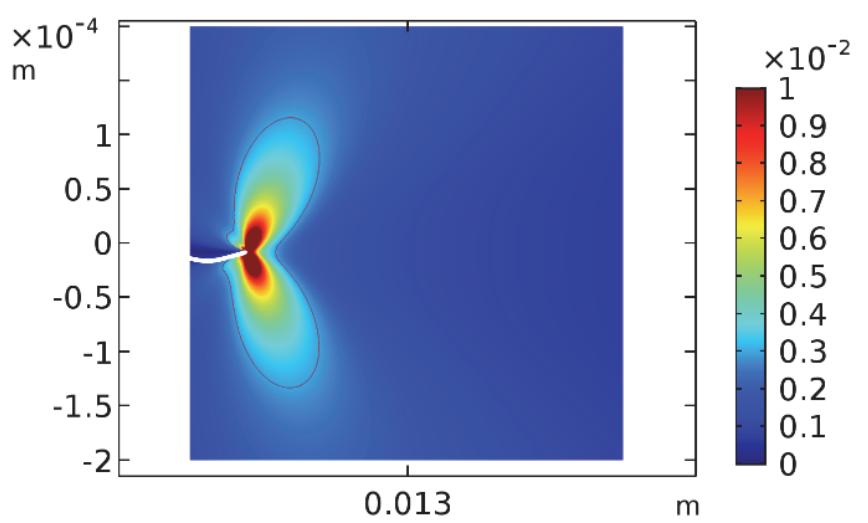

(b)

Figure 2: Plastic strain $\left(\eta=1\right.$, component $\left.\varepsilon_{i}\right)$ : (a) numerical simulation; (b) analytic solution by Eqn. (4).

The analysis of data presented in Fig. 3 allows us to conclude that the maximum relative error of Eqn. (1) is less than 20 percent. As a result, we can expect qualitative description of energy dissipation process at fatigue crack based on the simple hypothesis (4).

\section{EXPERIMENTAL STUDY OF STRAIN FIELD AT THE CRACK TIP}

A series of samples made of Grade 2 titanium alloy were tested using servo-hydraulic biaxial testing system Biss BI00-502, located in Kazan Scientific Center of Russian Academy of Sciences. The photo of the experimental setup is presented in Fig. 4. The geometry of the samples is shown in Fig. 5. During tests the samples were subjected to cyclic loading of $10 \mathrm{~Hz}$ with constant stress amplitude, different biaxial coefficient $\eta=\mathrm{Px} / \mathrm{Py}(1,0.7,0.5,0)$ and stress ratio $\mathrm{R}(0.1,0.3,0.5)$. The crack length was measured by an optic method.

The strain field was measured by digital image correlation method based on StrainMaster system and Lavision software. Surface of the sample near the crack tip was polished and covered with a black matte paint before the experiment. Then, the white paint was sprayed over the black paint to obtain a high contrast image. A macro lens and an elevated Led lamp were used for recording of material displacement at the crack tip. To restore the deformation field at the crack tip area, each frame was subjected to additional processing: calibration to level distortions caused by distortion of the lens, motion compensation, regulation of the illumination with digital filters. The spatial resolution of the strain field near the crack tip was 3e-6 m. Fig. 6 shows the characteristic result of measurements for the case of uniaxial loading. The deformation field is asymmetric due to the influence of the relative direction of the crack.

Fig. 7 shows the shape of the plastic area boundary and characteristic sizes which have been used for the comparison of the experimental and numerical data. Tab. 1 gives a quantitative comparison of the obtained data. 


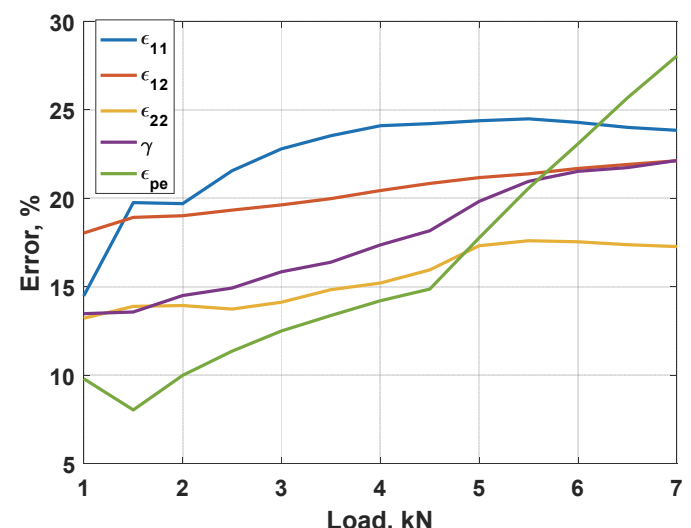

(a)

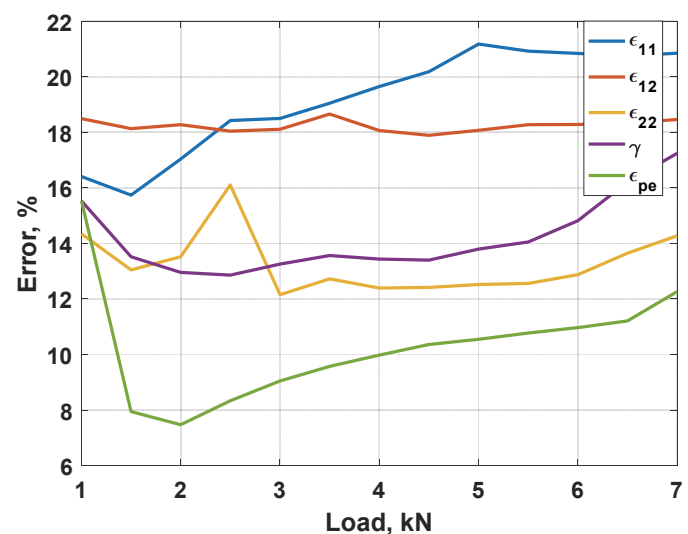

(c)

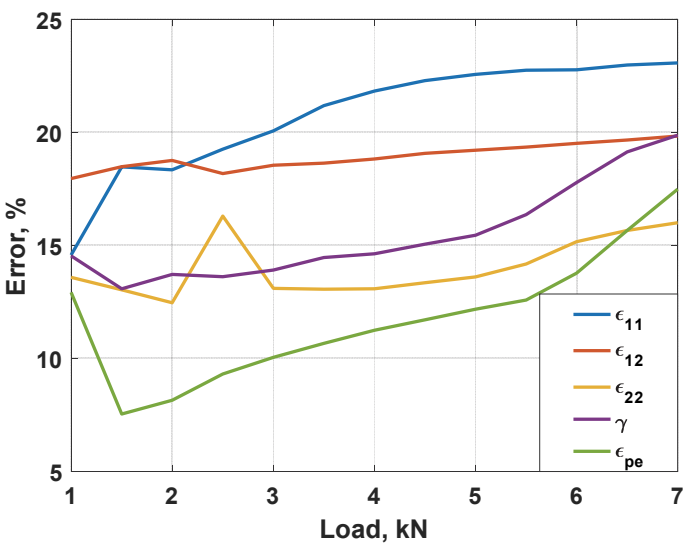

(b)

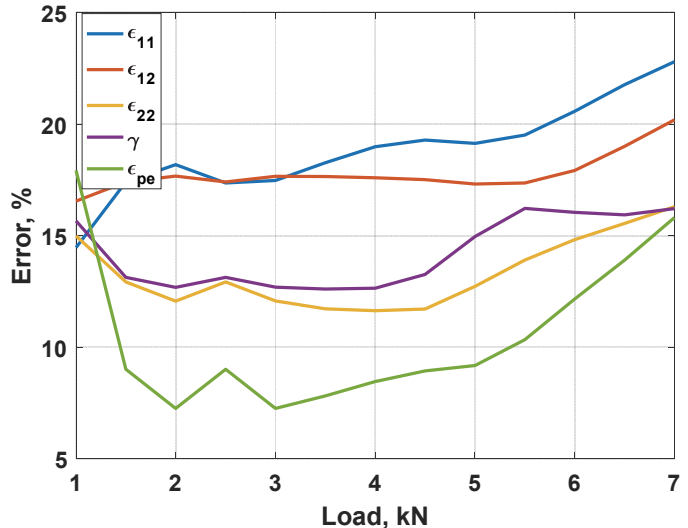

(d)

Figure 3: A relative error in plastic strain prediction (a) $\eta=0$; (b) $\eta=0.5$; (c) $\eta=0.7$; (d) $\eta=1$.

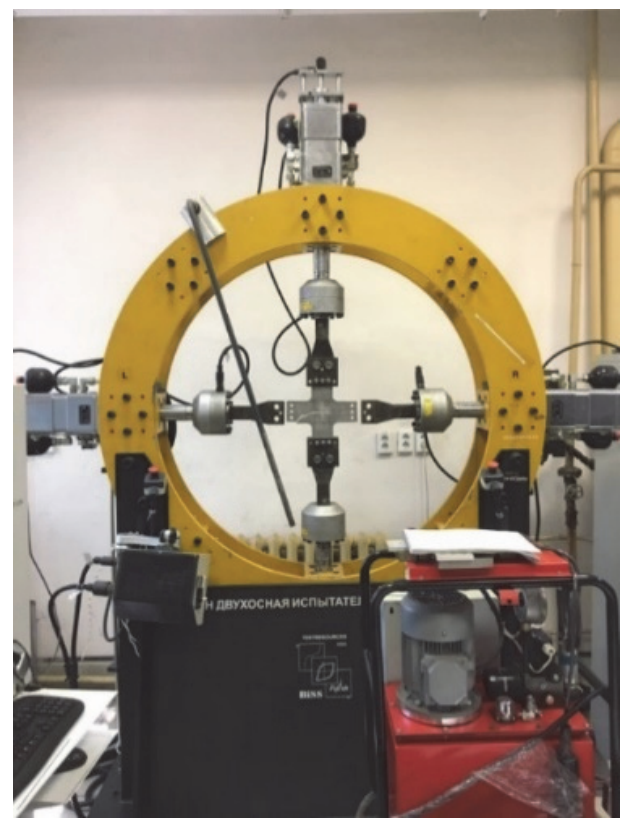

Figure 4: Testing machine Biss BI-00-502, Biaxial testing System.

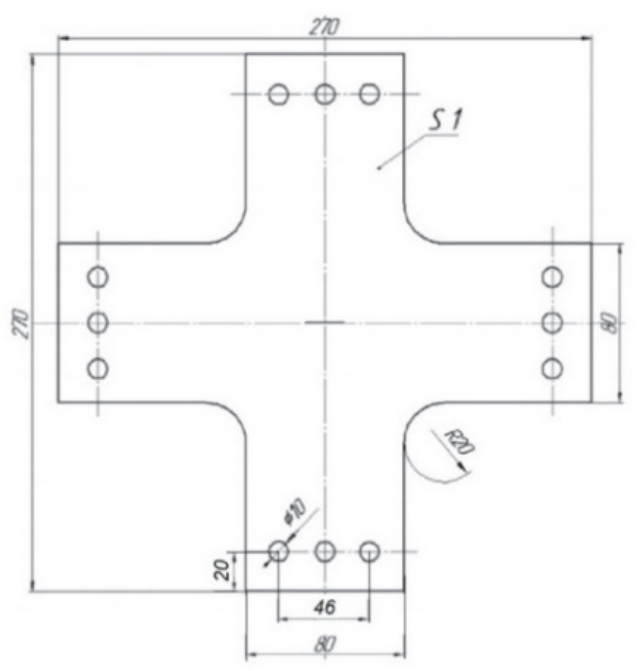

Figure 5: Geometry of samples (all sizes in millimeters). 


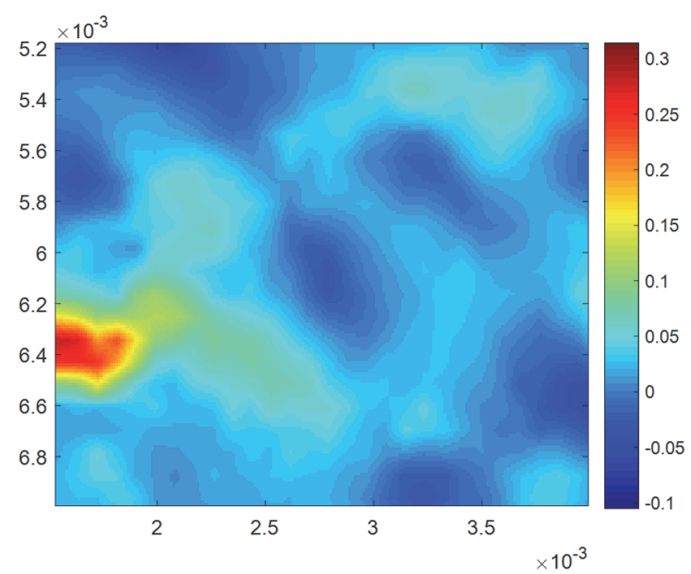

Figure 6: Measured field of strain for uniaxial loading

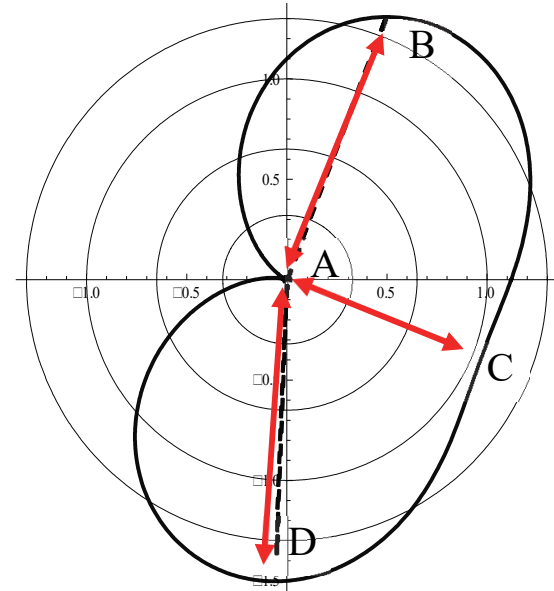

Figure 7: Boundary of plastic area.

\begin{tabular}{crc}
\hline & Numerical, $m m$ & Measure, $\mathrm{mm}$ \\
$\mathrm{AB}$ & $1,20 \mathrm{E}-01$ & $9,13 \mathrm{E}-02$ \\
$\mathrm{AC}$ & $1,35 \mathrm{E}-02$ & $3,13 \mathrm{E}-02$ \\
AD & $1,19 \mathrm{E}-01$ & $6,98 \mathrm{E}-02$ \\
BD & $2,18 \mathrm{E}-01$ & $1,27 \mathrm{E}-01$ \\
\hline
\end{tabular}

Table 1: Character size of plastic zone.

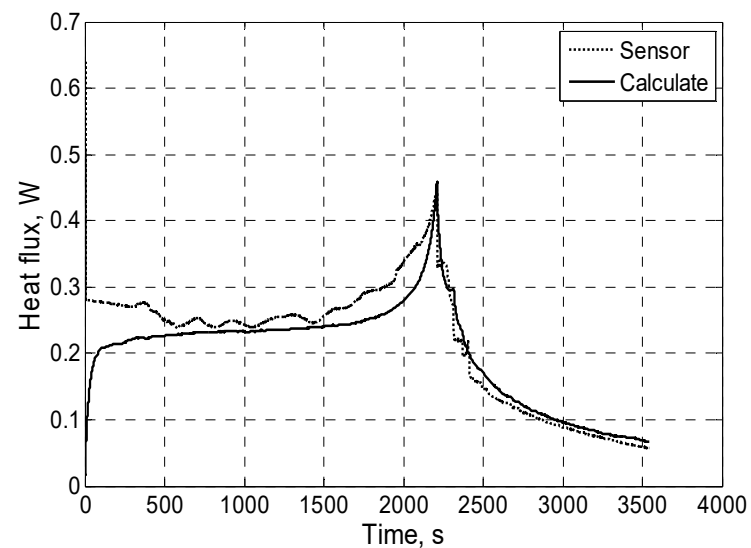

Figure 8: Heat dissipation histories obtained under constant stress amplitude (up to 2200-th second of the test) and constant stress intensity factor (remaining time) (Solid line approximation (1), the dotted line - experimental results).

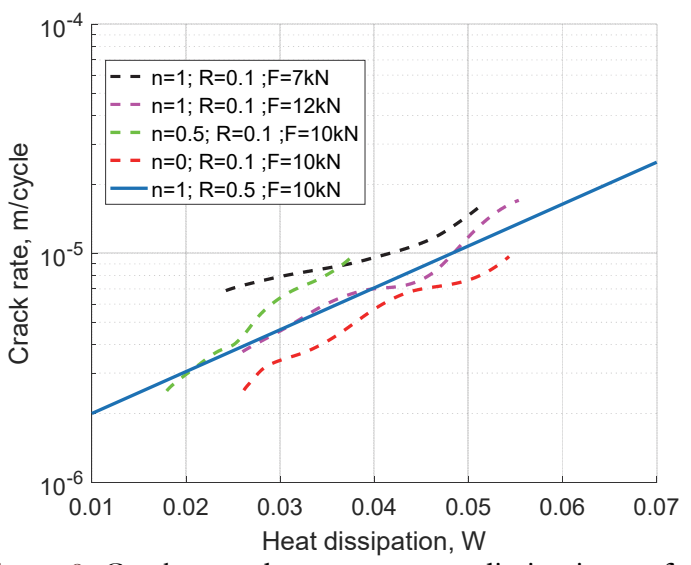

Figure 9: Crack growth versus energy dissipation at fatigue crack tip under biaxial loading

Details of heat measurement during the fatigue test can be find in [9]. The Eqn. (1) gives a good qualitative description of peculiarities of heat dissipation in both regimes with the constant stress amplitude and constant stress intensity factor, Fig. 8. Experimental works $[3,16]$ have shown that the rate of the stored energy has a maximum value on the initial stage of the plastic deformation. From the analysis of the energy balance, it follows that the dissipated energy during the cycle of the deformation differs from the expended energy insignificantly. We used the assumption of the proportionality of dissipated energy and plastic work. This formally corresponds a approximation of small stored energy. For constant stress amplitude the plastic work and, as a consequence, energy dissipation at the crack tip is determined by the crack growth rate as is shown [17] but for constant crack rate we can observe the regimes with the decrease of the heat dissipation caused by the decrease 
of the applied stress amplitude. The same comparison of experimental results and approximation (1) can be obtained for biaxial test (Fig. 9).

\section{CONCLUSION}

A series of experiments was carry out. For different biaxial coefficients, the strain field at the fatigue crack tip was measured. The results of the measurements have a good agreement with numerical simulation. The theoretical calculation of plastic deformation based on the elastic solution and the secant modulus of elasticity is carried out. According to the results of calculations, the error in determining the plastic deformation through the elastic solution does not exceed $30 \%$ for each component of the strain tensor, the maximum shear strain, and the intensity of plastic deformations.

This allows us to conclude that it is possible to use Eqn. (4) for the theoretical calculation of the deformation field at the fatigue crack tip and the subsequent calculation of energy dissipation.

An approximation for the energy dissipation at fatigue crack tip was proposed. The proposed phenomenological equation gives a good qualitative description of peculiarities of the heat dissipation. For the constant stress amplitude the plastic work and, as a consequence, energy dissipation at the crack tip is a linear function of the crack rate but for the constant crack rate mode the scenarios with a drop in the heat dissipation takes place. This approximation was confirmed by the experimental heat measurement from the crack tip under two experimental tests (constant stress amplitude and constant stress intensity factor) and different coefficients of biaxiality.

\section{ACKNOWLEDGMENTS}

he work was supported by the Russian Science Foundation (grant No. 19-77-30008).

\section{REFERENCES}

[1] Matvienko, Yu.G., Morozov, E M. (2004). Calculation of the energy J-integral for bodies with notches and cracks, International Journal of Fracture, 125, pp. 249-261.

[2] Rosakis, P., Rosakis, A.J., Ravichandran, G., Hodowany, J. A, (2000). Thermodynamic internal variable model for the partitional of plastic work into heat and stored energy in metals, J. Mech. Phys. Solids, 48, pp. 581-607.

[3] Oliferuk, W., Maj, M., Raniecki, B. (2004). Experimental Analysis of Energy Storage Rate Components during Tensile Deformation of Polycrystals, Materials Science and Engineering, 374, pp. 77-81.

[4] Izyumova, A., Plekhov, O. (2014). Calculation of the energy J-integral in plastic zone ahead of a crack tip by infrared scanning, FFEMS, 37, pp. 1330-1337.

[5] Meneghetti, G., Ricotta, M. (2016). Evaluating the heat energy dissipated in a small volume surrounding the tip of a fatigue crack, International Journal of Fatigue, 92(2), pp. 605-615.

[6] Risitano, A., Risitano, G. (2013). Cumulative damage evaluation in multiple cycle fatigue tests taking into account energy parameter, International Journal of Fatigue, 48, pp. 214-222.

[7] Ranganathan, N., Chalon, F., Meo, S. (2008). Some aspects of the energy based approach to fatigue crack propagation Original research article, International Journal of Fatigue, 30, pp. 1921-1929.

[8] Fedorova, A.Yu., Bannikov, M.V., Terekhina, A.I., Plekhov, O.A. Heat dissipation energy under fatigue based on infrared data processing (2014). Quantitative InfraRed Thermography Journal, 11(1), pp. 2-9

[9] Vshivkov, A., Iziumova, A., Bär, U., Plekhov, O. (2016). Experimental study of heat dissipation at the crack tip during fatigue crack propagation, Frattura ed Integrità Strutturale, 35, pp. 131-137.

[10] Raju, K.N. (1972). An energy balance criterion for crack growth under fatigue loading from considerations of energy of plastic deformation, International Journal of Fracture Mechanics, 8(1), pp. 1-14.

[11] Dixon, J.R. (1965). Stress and strain distributions around cracks in sheet materials having various work-hardening characteristics, Ministry of Technology, National Engineering Laboratory, Materials Group: East Kilbride, Glasgow, Scotland, pp. 224-244. 
[12] Hammouda M.M.I., Fayed A.S., Sallam H.E.M. (2004). Stress intensity factors of a central slant crack with frictional surfaces in plates with biaxially loading, Int. J.Fract, 129, pp. 141-148.

[13] Hammouda M.M.I., Fayed A.S., Sallam H.E.M. (2003). Simulation of mixed mode I/II cyclic deformation at the tip of a short kinked inclined crack with frictional surfaces. J. of Fatigue, 25(8), pp. 743-753.

[14] Hammouda M.M.I., Fayed A.S., Sallam H.E.M. (2003). Stress intensity factors of a shortly kinked slant central crack with frictional surfaces in uniaxially loaded plates, J. of Fatigue, 25(4), pp. 283-298.

[15] Hammouda M.M.I., Fayed A.S., Sallam H.E.M. (2002). Mode II stress intensity factors for central slant cracks with frictional surfaces in uniaxially compressed plates, J. of Fatigue, 24(12), pp. 1213-1222.

[16] Bever M.B., Holt D.L., Titchener A.L. (1973). The stored energy of cold work, Progress in Materials Science, 17, pp. 5177.

[17] Iziumova A., Vshivkov A., Plekhov O. (2016). The study of the effect of energy dissipation at fatigue crack tip on its propagation rate. 13th Quantitative Infrared Thermography Conference. Gdańsk, Pologne, paper 101. 